\title{
Text Messages Can Encourage Patients to Discuss and Receive HIV Testing in Primary Care
}

\author{
Renata Wettermann, BA, Haley Marek, BS, Thomas P. Giordano, MD, MPH, and \\ Monisha Arya, MD, MPH
}

Objective: Routine HIV testing is not adequately occurring in primary care. One reason is that physicians perceive that patients do not want to discuss HIV testing and would prefer that patients initiate the discussion. A patient-centered text message campaign may prompt patients to discuss HIV testing with their physicians, thereby increasing HIV testing.

Methods: The study took place in clinics serving low-income populations. Participants received a randomized text message 30 minutes before their appointment, prompting them to discuss either HIV or an unrelated health topic with their physician. Participants were interviewed after their appointment to ascertain if they had discussed HIV testing, and test orders were verified via electronic medical record.

Results: Among participants sent an HIV text message $(n=17), 6$ were HIV tested (35\%). No participants sent a control text message were HIV tested. of the 10 participants who reported reading the HIV message, 7 (70\%) discussed HIV with their physician and $6(60 \%)$ were tested.

Conclusion: Our proof-of-concept study suggests an HIV text message campaign may increase HIV testing by encouraging patients to initiate discussion with their physicians. This intervention may increase HIV testing among low-income populations. A larger study is needed to confirm these findings. (J Am Board Fam Med 2019;32:408-412.)

Keywords: AIDS, Low-Income Population, Patient Activation, Physicians, Primary Health Care, Proof of Concept Study, Telemedicine, Text Messaging

A striking $40 \%$ of new HIV infections in the US may be attributed to the $16 \%$ of HIV-positive individuals who remain unaware of their status because they have not been tested. ${ }^{1}$ Because of this threat to personal and public health, the US Centers for Disease Control and Prevention (CDC) made a major revision to their HIV testing recommendations in

This article was externally peer reviewed.

Submitted 11 September 2018; revised 6 January 2019; accepted 8 January 2019.

From the School of Medicine, Baylor College of Medicine, Houston, TX (RW, HM); Department of Medicine, Baylor College of Medicine (TPG, MA); Center for Innovations in Quality, Effectiveness and Safety, Michael E. DeBakey VA Medical Center, Houston, TX (TPG, MA).

Funding: This work was supported by a Baylor College of Medicine-University of Texas Houston Center for AIDS Research grant (Grant 3 P30 AI 03621120 S1). This work was supported in part by the Houston Center for Innovations in Quality, Effectiveness and Safety, Michael E. DeBakey Veterans Affairs Medical Center.

Conflict of interest: none declared.

Corresponding author: Renata Wettermann, BA, Baylor College of Medicine, 1 Baylor Plaza, Houston, TX 77030 (E-mail: wetterma@bcm.edu).
2006. In contrast to prior testing recommendations, the CDC now recommends that all Americans ages 13 to 64 years be screened for HIV as part of routine medical care regardless of risk factors. ${ }^{2}$ However, the CDC reports that only $39.6 \%$ of adults have ever been tested, ${ }^{3}$ indicating a lack of routine screening. Due to delayed testing, low-income adults commonly receive a late HIV diagnosis ${ }^{4,5}$ putting them at risk for rapidly developing AIDS.

Primary care physicians face many barriers to implementing HIV testing, and as a result patients are not being routinely screened for HIV. ${ }^{1}$ DeRose et al ${ }^{6}$ determined that nearly a third of patients with an eventual HIV diagnosis had missed opportunities for testing during physician appointments. A challenge hampering routine screening is that physicians perceive that patients do not want or need a test. ${ }^{7}$ Accordingly, one study found that $71 \%$ of physicians would prefer their patient ask for an HIV test instead of broaching the subject themselves. ${ }^{8}$ Patients would be comfortable asking their physician for an HIV test ${ }^{9}$ but are unsure how to do so. ${ }^{10}$ Therefore, an 
intervention to prompt patients to ask for an HIV test may increase testing in primary care.

Patient education interventions delivered immediately before primary care appointments can prompt patients to communicate with their physicians. ${ }^{11}$ Prompts delivered via text message can engage patients in their health, ${ }^{12}$ are preferred by low-income adults, ${ }^{13}$ and can be sent in a time-sensitive manner (for example, minutes before an appointment). We developed a text message campaign to educate patients about HIV testing and encourage them to discuss testing with their physician. The purpose of this proof of concept study was to assess the feasibility of using a patient-facing text message campaign to facilitate HIV testing discussions and increase HIV testing in primary care.

\section{Methods}

\section{Study Location}

This study took place from March to July 2016 in 2 urban family medicine clinics with 19 physicians within the Harris Health System (Harris Health), the largest publicly funded health care system serving predominately low-income patients in Houston, TX.

\section{Text Message Content}

Research staff created 11 draft HIV testing text messages with content that attempted to address known barriers and facilitators to HIV testing. Because audience segmentation is critical for message targeting, a convenience sample of Harris Health patients within the majority age demographic of the clinic population (ages 35 to 55 years) was selected to review the draft text messages. These 10 patients helped ensure understanding and acceptability of the text message content. After receiving input from patients, the research staff finalized 4 HIV testing text messages. Each message was personalized to include the participant's name and physician's name. Messages included a fact to educate patients about HIV testing and a prompt such as "when you see Dr. ' $\mathrm{X}$ ', say: Please test me for HIV today." There were 2 control messages discussing vaccines and calcium intake, which were also personalized and included a prompt (eg, "remind Dr. X to check if you are due for any vaccines today"). (See Figure 1 for the themes of the finalized messages.)

\section{Recruitment}

Harris Health information technology staff prescreened patients according to the following criteria: ages 35 to 55 years, English speaking, no prior HIV test in the electronic medical record (EMR), and with an upcoming primary care appointment in the 2 participating clinics. Research staff called eligible patients to explain the study and participant responsibilities, which included receiving a health tip via text message and participating in an evaluation interview. For interested individuals, research staff confirmed eligibility and obtained verbal consent. Only patients who reported routinely carrying a cell phone with text messaging capabilities and planning to attend their appointment were enrolled. Participants who completed the evaluation interview were offered a $\$ 10$ gift card. Physicians were not made aware of the study to avoid altering their behavior. The Baylor College of Medicine Institutional Review Board approved this study.

\section{Text Message Delivery and Evaluation}

Thirty minutes before a participant's appointment time, research staff sent a randomized study text message to the participant's cell phone. About 1 hour after the participant's appointment, research staff called the participant for an evaluation interview. Participants were asked if they had received the message and read it before their appointment. In addition, they were asked if they had communicated with their physician about vaccines, calcium, and HIV. Following the interview, a ret-

Figure 1. Human immunodeficiency virus (HIV) and control text message themes.

\begin{tabular}{|c|l|}
\hline Text Message Type & \multicolumn{1}{c|}{ Text Message Theme } \\
\hline HIV Awareness & All adult patients are tested for HIV at Harris Health. \\
\hline HIV Instruction & Ask your doctor for an HIV test. \\
\hline $\begin{array}{c}\text { HIV Positive } \\
\text { Persuasive }\end{array}$ & HIV is asymptomatic; Testing is necessary for diagnosis. \\
\hline $\begin{array}{c}\text { HIV Negative } \\
\text { Persuasive }\end{array}$ & $\begin{array}{l}\text { HIV-infected persons who do not know their status can give HIV to } \\
\text { their partners unknowingly. }\end{array}$ \\
\hline Calcium Control & Adults need 1,000 milligrams of calcium per day. \\
\hline Vaccine Control & There are vaccines that even adults need. \\
\hline
\end{tabular}


rospective EMR review was performed to ensure that the participant had attended their appointment, collect demographic information, and see if an HIV test was ordered. Using the EMR, we assessed whether or not the HIV test was ordered on the day the text message was sent to assess physician response to patient prompt. Participants who did not attend their appointment were excluded from analysis.

\section{Data Analysis}

Descriptive statistics were used to summarize participant demographics. Testing ordering rates were calculated from EMR data. The proportion of participants who read the study message and discussed HIV was obtained from evaluation interview responses.

\section{Results}

Out of 136 patients who met eligibility criteria and were called for recruitment, 66 answered the phone, and 36 consented and enrolled. One participant was not sent a text message due to a research team error, and 6 participants did not attend their scheduled appointment. As a result, there were 29 evaluable participants.

Among these 29 participants, 21 (72\%) were female and 8 (28\%) were male. The racial and ethnic distribution of participants was 9 Hispanic/ Latino (31\%), 10 Black/African American (34\%), 9 white $(31 \%)$, and 1 Asian (3\%). All participants were in their forties (45\%) or fifties (55\%), with an average age of 49 years.

Of the 17 participants who received an HIVrelated text message, EMR review found $6(35 \%)$ had an HIV test ordered on the day the message was sent. In contrast, none of the 12 participants who received the control message had an HIV test ordered.

As illustrated in Figure 2, 23 participants (79\%) completed an evaluation interview. Sixteen participants $(70 \%)$ reported reading the text message, of whom 10 received the HIV message and 6 received the control message. Of those that read the HIV text message, 7 (70\%) said they discussed HIV testing with their physician and $6(60 \%)$ had an HIV test ordered. In comparison, none of the participants who received a control message reported discussing HIV with their physician or had an HIV test ordered.

\section{Discussion}

In this proof-of-concept study, we found that 35\% of participants who received an HIV text message had an HIV test ordered, compared with none of the control group participants. Furthermore, among participants who confirmed reading the HIV text message before their appointment, $60 \%$ had an HIV test ordered compared with none of the participants who reported not reading the message. This suggests that the text message served as a cue to action for patients to initiate discussion about HIV testing. These positive results may be attributed to our campaign's use of personalization and a cue to action. Research has demonstrated that personalization increases the impact of campaigns, ${ }^{12}$ while a cue to action is a fundamental element of the Health Belief Model of Behavior Change. ${ }^{14}$

Interestingly, 2 participants who had an HIV test ordered reported reading the message but not discussing HIV with their physicians. It is possible that these participants had a discussion with their physician about HIV testing but felt uncomfortable disclosing it to the research team. Alternatively, the physician may have offered an HIV test; nevertheless, the text message may have still played a role in increasing acceptability of HIV testing even among participants who were not yet ready to ask for a test themselves.

In this study of low-income patients, all the participants who were HIV tested were over 40 years old, and $83 \%$ were women. Our findings are relevant as one third of new HIV diagnoses are among people above the age of 40 years, ${ }^{15}$ yet this age-group is less frequently tested for HIV. ${ }^{16} \mathrm{HIV}$ testing rates are also particularly low among older women. ${ }^{17}$ In addition, low-income communities face higher HIV prevalence, later HIV diagnosis, and more rapid progression to AIDS following diagnosis. $^{5}$

Generalizability was a limitation of this proof of concept study. This study involved only patients ages 35 to 55 years who were English speaking, reflecting the demographics of the patients who had pretested our messages. Our future work will focus on expansion to include all ages and nonEnglish speaking patients to better adhere to the CDC recommendations for HIV testing. As participants opted into the study, there may have been selection bias toward more proactive patients. It is 
Figure 2. Participant self-reported communication and EMR-verified human immunodeficiency virus (HIV) test orders following an HIV text message campaign. EMR, electronic medical record.

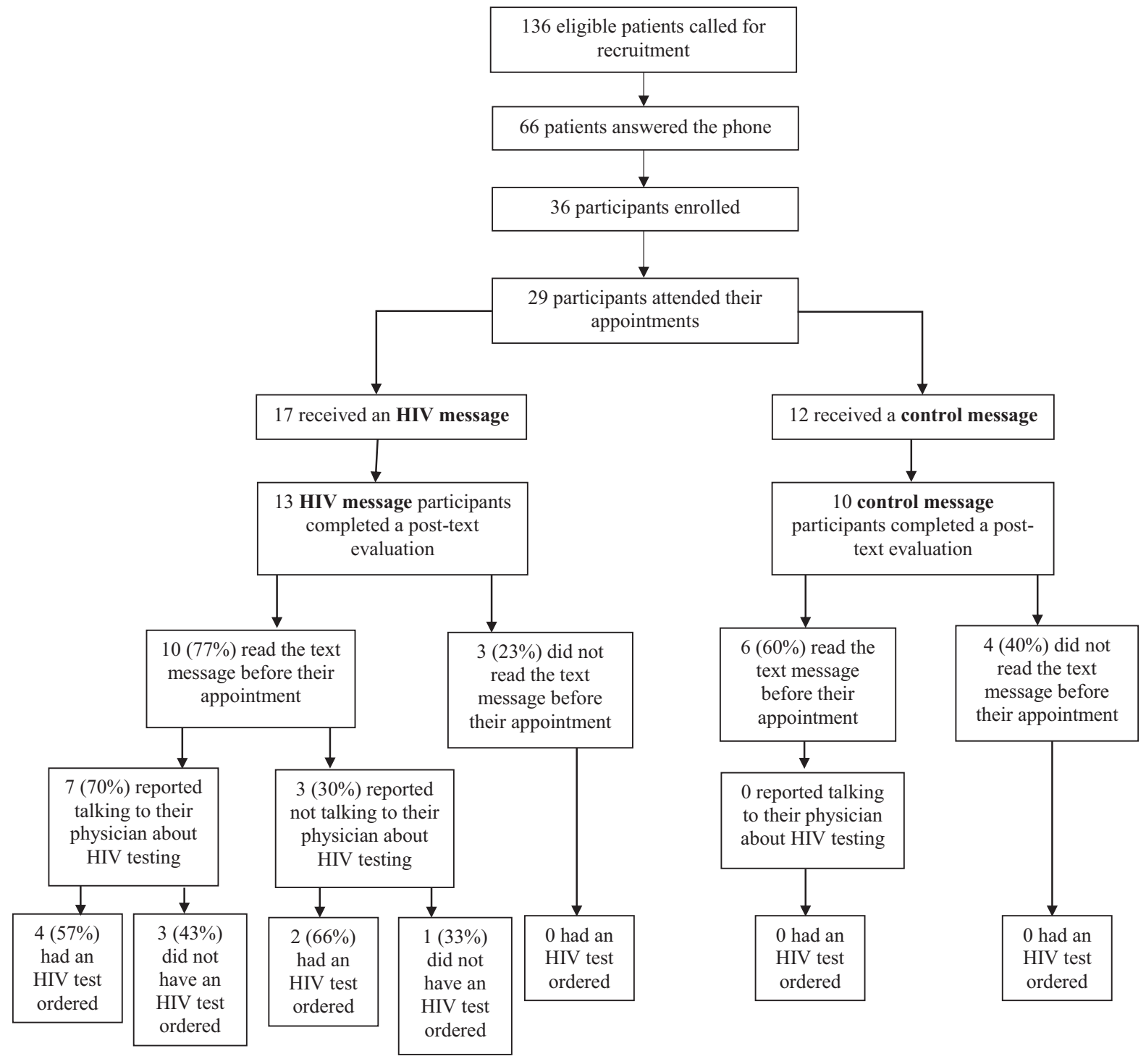

possible that text message timing was not optimal. Some participants may have not had the opportunity to read the text message until after their appointment, at which point it was too late to initiate an HIV testing discussion. Another limitation was the reliance on self-reported data to determine if participants had read the message and talked about HIV testing. Self report may be influenced by social desirability bias. ${ }^{18}$ In addition, we could not assess the $21 \%$ of participants who did not complete the evaluation interview. In future work, we hope to request a secondary method of contact to reduce loss to followup. Finally, because this was a proof of concept study, HIV testing rates in the clinics were not compared before and after the intervention, but would be an important consideration in larger studies of text message campaigns. Despite these limitations, our HIV text message campaign proof of concept study showed promise as a tool to increase HIV status awareness by enhancing communication between patients and their physicians.

\section{Conclusion}

A personalized and timely text message that prompts patients to ask for an HIV test may complement physician-facing initiatives (eg, EMR alerts) to improve HIV testing. Patients' requests have a significant impact on physicians' orders, ${ }^{18}$ 
suggesting that patients who ask for an HIV test may receive it. Our proof of concept study demonstrates the feasibility of using customized text messages to encourage patient-physician communication about HIV testing discussions, and highlights that this strategy can improve HIV testing in primary care. While a larger study is needed to confirm our findings, a patient-facing HIV testing text message campaign may be one strategy to address the disproportionate burden of HIV in low-income settings.

Our sincere thanks to Stephen Manning for assistance in finalizing text message content and performing data collection, to Sharon Joy for her assistance with data collection, to Rebecca Fisk for her thoughtful input throughout the writing process, and to Disha Kumar for her role in conceptualizing this study.

To see this article online, please go to: http://jabfm.org/content/ 32/3/408.full.

\section{References}

1. Dailey AF. Vital signs: Human immunodeficiency virus testing and diagnosis delays-United States. MMWR Morb Mortal Wkly Rep 2017;66: 1300-6.

2. Revised recommendations for HIV testing of adults, adolescents, and pregnant women in health-care settings. Available from: https://www.cdc.gov/mmwr/ preview/mmwrhtml/rr5514a1.htm. Accessed July 6, 2018.

3. Pitasi MA. Interval since last HIV test for men and women with recent risk for HIV infection-United States, 2006-2016. MMWR Morb Mortal Wkly Rep 2018;67:677-81.

4. Centers for Disease Prevention and Control. CDC fact sheet: Today's HIV/AIDS epidemic. August 2016. Available from: https://www.cdc.gov/nchhstp/ newsroom/docs/factsheets/todaysepidemic-508.pdf. Accessed December 17, 2018.

5. Krawczyk CS, Funkhouser E, Kilby JM, Vermund SH. Delayed access to HIV diagnosis and care: Special concerns for the Southern United States. AIDS Care 2006;18(Suppl 1):S35-S44.

6. DeRose J, Zucker J, Cennimo D, Swaminathan S. Missed testing opportunities for HIV screening and early diagnosis in an urban tertiary care center. AIDS Res Treat 2017;2017:5708620.
7. White BL, Walsh J, Rayasam S, Pathman DE, Adimora AA, Golin CE. What makes me screen for HIV? Perceived barriers and facilitators to conducting recommended routine HIV testing among primary care physicians in the Southeastern United States. J Int Assoc Provid AIDS Care 2015;14:127-35.

8. Arya M, Patel S, Kumar D, et al. Why Physicians don't ask interpersonal and intrapersonal barriers to HIV testing-Making a case for a patient-initiated campaign. J Int Assoc Provid AIDS Care 2016;15: $306-12$.

9. Arya M, Huang A, Kumar D, Hemmige V, Street RL, Giordano TP. The promise of patient-centered text messages for encouraging HIV testing in an underserved population. J Assoc Nurses AIDS Care 2018;29:101-6.

10. 2009 Survey of Americans on HIV/AIDS. Available from: https://www.kff.org/hivaids/poll-finding/2009survey-of-americans-on-hivaids-toplines/. Accessed July 26, 2018.

11. Deen D, Lu WH, Rothstein D, Santana L, Gold MR. Asking questions: The effect of a brief intervention in community health centers on patient activation. Patient Educ Couns 2011;84:257-60.

12. Head KJ, Noar SM, Iannarino NT, Grant Harrington $\mathrm{N}$. Efficacy of text messaging-based interventions for health promotion: A meta-analysis. Soc Sci Med 2013;97:41-8.

13. Patel S, Hemmige V, Street RL, Viswanath K, Arya M. Activating Racial and Ethnic Minorities to Engage in Preventive Health: Patient Preferences for Health Reminders. J Particip Med 2017;9. pii: e8 [Epub ahead of print.]

14. Hayden JA. Introduction to Health Behavior Theory. Burlington, MA: Jones \& Bartlett Publishers; 2013.

15. Centers for Disease Control and Prevention. HIV among people aged 50 and over. May 15, 2018. Available from: https://www.cdc.gov/hiv/group/age/ olderamericans/index.html. Accessed July 26, 2018.

16. Ham DC, Lecher S, Gvetadze R, Huang LA, Peters P, Hoover KW. HIV testing at visits to physicians' offices in the U.S., 2009-2012. Am J Prev Med 2017;53:634-45.

17. Guo Y, Sims OT. Assessment of recent HIV testing among older adults in the United States. Soc Work Health Care 2017;56:855-64.

18. Bowling A. Mode of questionnaire administration can have serious effects on data quality. J Public Health (Oxf) 2005;27:281-91.

19. Kravitz RL, Epstein RM, Feldman MD, et al. Influence of patients' requests for direct-to-consumer advertised antidepressants: A randomized controlled trial. JAMA 2005;293:1995-2002. 